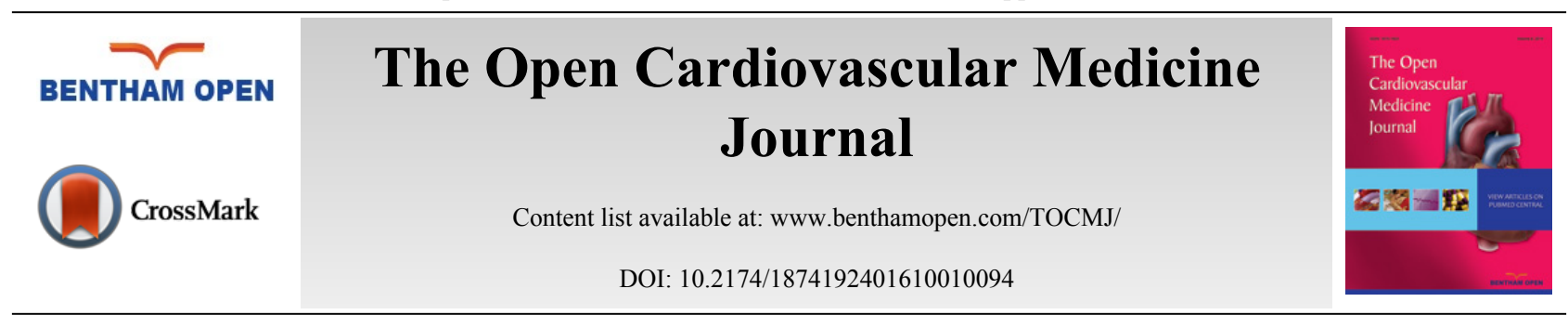

\title{
Newer Oral Anticoagulants: Stroke Prevention and Pitfalls
}

\author{
Anand Patel ${ }^{1, *}$, Richard P. Goddeau $\mathrm{Jr}^{1}$ and Nils Henninger ${ }^{1,2}$ \\ ${ }^{I}$ Department of Neurology, University of Massachusetts Medical School, Worcester, MA, USA \\ ${ }^{2}$ Department of Psychiatry, University of Massachusetts Medical School, Worcester, MA, USA
}

Received: September 29, 2015

Revised: October 08, 2015

Accepted: November 25, 2015

\begin{abstract}
Warfarin is very effective in preventing stroke in patients with atrial fibrillation. However, its use is limited due to fear of hemorrhagic complications, unpredictable anticoagulant effects related to multiple drug interactions and dietary restrictions, a narrow therapeutic window, frequent difficulty maintaining the anticoagulant effect within a narrow therapeutic window, and the need for inconvenient monitoring. Several newer oral anticoagulants have been approved for primary and secondary prevention of stroke in patients with non-valvular atrial fibrillation. These agents have several advantages relative to warfarin therapy. As a group, these direct oral anticoagulants (DOAC), which include the direct thrombin inhibitor, dabigatran, and the factor Xa inhibitors (rivaroxaban, apixaban, and edoxaban), are more effective than dose adjusted warfarin for prevention of all-cause stroke (including both ischemic and hemorrhagic stroke), and have an overall more favorable safety profile. Nevertheless, an increased risk of gastrointestinal bleeding (with the exception of apixaban), increased risk for thrombotic complication with sudden discontinuation, and inability to accurately assess and reverse anticoagulant effect require consideration prior to therapy initiation, and pose a challenge for decision making in acute stroke therapy.
\end{abstract}

Keywords: Atrial fibrillation, hemorrhage, ischemic stroke, oral anticoagulation, outcome, review, therapy, thrombolysis.

\section{INTRODUCTION}

Until recently vitamin $\mathrm{K}$ antagonists such as warfarin were the only available oral anticoagulants for stroke prevention in patients with atrial fibrillation. Warfarin is very effective in preventing stroke in patients with atrial fibrillation with an approximately two-thirds reduction in stroke incidence on the basis of a meta-analysis of the randomized controlled trials [1]. However, it is often regarded as suboptimal [2] partly due to its pharmacological properties, which include unpredictable anticoagulant effects, genetic variability in metabolism, multiple drug interactions and dietary restrictions, a narrow therapeutic window, and the resulting need for inconvenient monitoring [3]. In addition, warfarin carries a significant risk for hemorrhagic complications including intracranial hemorrhages [4 $-6]$.

Since 2010, several novel oral anticoagulants (NOAC) have been approved for use in the United States by the federal Food and Drug Administration (FDA) for primary and secondary prevention of stroke in patients with nonvalvular atrial fibrillation. These agents hold promise to overcome several of the limitations associated with Warfarin therapy. NOAC, as a group, presently include the direct thrombin inhibitor dabigatran and the factor Xa inhibitors rivaroxaban, apixaban and edoxaban. While the acronym NOAC is being used to denote their novelty over warfarin, alternate naming such as direct oral anticoagulants (DOAC) may be preferred in the future, particularly in light of expected further developments in the field of oral anticoagulants.

In the following we will provide a brief summary of the trials investigating oral DOACs for the treatment of atrial fibrillation, advantages and disadvantages of their use over warfarin, and highlight areas of uncertainty with respect to their use.

\footnotetext{
* Address correspondence to this author at the Department of Neurology, University of Massachusetts Medical School, 55 Lake Ave, North, Worcester, MA 01655, USA; Tel: (508) 334-2527; Fax: (508) 856-6778; Email: Anand.Patel@umassmemorial.org
} 


\section{DIRECT THROMBIN INHIBITOR}

\section{Dabigatran}

Dabigatran etexilate is a pro-drug that is rapidly converted by a serum esterase to dabigatran which is a direct and competitive inhibitor of thrombin (factor IIa). Dabigatran has predictable pharmacokinetics allowing for a fixed dose regimen twice daily due to its elimination half-life of $12-17$ hours. Because $80 \%$ is renally excreted, baseline renal function affects its pharmacokinetics. While dabigatran is not metabolized via the cytochrome P450 system, several p-glycoprotein inhibitors such as verapamil, amiodarone, ketoconazole, dronedarone, quinidine can decrease its metabolism potentially leading to increased bleeding complications related to its use, conversely rifampin can increase its metabolism [7].

Its clinical efficacy in preventing ischemic stroke among patients with atrial fibrillation was evaluated in the Randomized Evaluation of Long-Term Anticoagulation (RE-LY) trial [8]. RE-LY was a non-inferiority trial comparing two different doses of dabigatran (110 mg and $150 \mathrm{mg}$ twice daily) to dose adjusted warfarin (goal INR 2.0-3.0) in patients with atrial fibrillation and at least one additional stroke risk factor (previous history of stroke or transient ischemic attack, a left ventricular ejection fraction of $\leq 40 \%$, New York Heart Association class $\geq 2$ heart failure with symptoms within 6 months prior to screening, and age of at least 75 years or an age of 65 to 74 years plus diabetes, hypertension or coronary artery disease). Patients with severe valvular heart disease, who had a stroke within 14 days or severe stroke within the 6 months prior to screening, a condition associated with increased hemorrhage risk, creatinine clearance of $<30 \mathrm{ml} / \mathrm{min}$, active liver disease, and pregnancy were excluded. The trial included 18113 patients with median follow up duration of 2 years. In the following only the key results for the $150 \mathrm{mg}$ dosing regimen are discussed as the $110 \mathrm{mg}$ dose has not been FDA approved in the United States.

Dabigatran, when administered at $150 \mathrm{mg}$ twice a day dosing, was more effective than warfarin in reducing the composite end point of stroke (defined as acute onset of focal neurological deficits respecting vascular territory and categorized as ischemic, hemorrhagic or unspecified strokes and hemorrhagic transformation of the ischemic stroke was not considered a hemorrhagic stroke) and systemic embolism (defined as acute vascular occlusion of an extremity or an organ documented by meaning of imaging, surgery or autopsy) by almost $36 \%(1.11 \% \mathrm{vs} .1 .69 \%$ per year; RR 0.66 , 95\% CI 0.53-0.82, $P<0.001)$. Importantly, there were significantly fewer ischemic strokes in patients receiving dabigatran as compared to warfarin $(0.92 \% v s .1 .20 \%$ per year; RR $0.76,95 \%$ CI $0.60-0.98, P=0.03)$. Along with a reduction in the incidence of ischemic stroke, there were also fewer hemorrhagic strokes in the dabigatran group $(0.10 \%$ vs. $0.38 \%$ per year; RR $0.26,95 \%$ CI $0.14-0.49, P<0.001)$. At the same time, the rate of myocardial infarction was higher in patients with receiving dabigatran than warfarin $(0.74 \% v s .0 .53 \%$ per year, $P=0.048)$ suggesting that dabigatran may be less effective in reducing cardiac complications than warfarin.

Major bleeding complications were similar in both the groups (3.11\% per year in dabigatran groups vs. $3.36 \%$ per year in warfarin group; RR 0.93, $P=0.31$ ). Patients receiving dabigatran had more gastrointestinal hemorrhages than warfarin $(1.51 \%$ vs. $1.02 \%$ per year; RR $1.50,95 \%$ CI $1.19-1.89, P<0.001)$ but at the same time, life threatening hemorrhages were less common in patients receiving dabigatran $(1.45 \%$ vs. $1.80 \%$ per year; RR $0.81,95 \% \mathrm{CI}$ 0.66-0.99, $P=0.04)$. There were fewer major and minor systemic hemorrhages noted among patients receiving dabigatran. The most feared complication of anticoagulant use-intracranial hemorrhage (defined as hemorrhagic stroke, subarachnoid hemorrhage and subdural hemorrhage)-was significantly lower in the dabigatran as compared to the warfarin arm (0.30\% vs. $0.74 \%$ per year; RR $0.40,95 \%$ CI $0.27-0.60, P<0.001)$.

Overall, dabigatran conferred a net clinical benefit (defined as a composite of stroke, systemic embolism, pulmonary embolism, myocardial infarction, major bleeding and death) over warfarin in the study population $(6.91 \%$ vs. $7.64 \%$ per year; RR $0.91,95 \%$ CI $0.82-1.00, P=0.04)$.

\section{Factor Xa Inhibitors}

Factor Xa inhibitors act by competitively binding to active site of activated factor X and thus inhibiting factor Xa. All factor Xa inhibitors have relatively few drug-drug interactions, except with CYP3A4 and p-glycoprotein pathway inhibitors, which could lead to higher anticoagulation effect than expected.

\section{Apixaban}

Apixaban has a rapid oral absorption and maximum plasma concentration is achieved in about 3 hours. Its half-life 
of 12 hours requires twice a day dosing. Steady state plasma concentrations are achieved after 3 days of regular use. It is mainly metabolized by the liver and approximately $75 \%$ are excreted in feces and the remaining $25 \%$ through kidneys $[9,10]$.

\section{Apixaban vs. Warfarin}

Apixaban for Reduction In STroke and Other ThromboemboLic Events in atrial fibrillation (ARISTOTLE) [11] was a randomized, double blind trial comparing apixaban $5 \mathrm{mg}$ twice a day dosing $(2.5 \mathrm{mg}$ twice a day dosing was used in patients with two or more of the following criteria: age more than 80 years, weight less than $60 \mathrm{~kg}$ or serum creatinine $\geq 1.5$ ) to warfarin (target INR 2.0-3.0) in patients with atrial fibrillation and one additional vascular risk factor for stroke (age $\geq 75$ years, history of prior stroke, transient ischemic attack or systemic embolism, congestive heart failure or left ventricular ejection fraction $\leq 40 \%$ and history of diabetes mellitus or hypertension requiring pharmacological treatment) [11]. The trial included 18201 patients with median follow up of 1.8 years.

Overall, a significant $21 \%$ relative reduction in the composite endpoint of all-cause stroke (defined as a focal neurological deficits from a non-traumatic cause lasting for at least 24 hours and including ischemic, with and without hemorrhagic transformation, hemorrhagic and unspecified strokes) and systemic embolism were noted in the apixaban arm compared to the warfarin arm $(1.27 \%$ vs. $1.60 \%$ per year; HR $0.79,95 \%$ CI $0.66-0.95, P=0.01$ for superiority). A substantial part of the benefit was governed by a greater (almost $50 \%$ relative) reduction in the hemorrhagic stroke in the apixaban group ( $0.24 \%$ vs. $0.47 \%$ per year; HR $0.51,95 \%$ CI $0.35-0.75, P<0.001)$; the rate of ischemic and other unspecified types of strokes were equal in both groups (respective $0.97 \% v s .1 .05 \%$ in apixaban $v s$. warfarin groups per year; HR $0.92,95 \%$ CI $0.74-1.13, P=0.42$ ). There was no significant difference in incidence of myocardial infarction, deep vein thrombosis, or pulmonary embolism between treatment arms.

Importantly, major bleeding as defined by the International Society on Thrombosis and Hemostasis (ISTH) was reduced in the apixaban group $(2.13 \%$ vs. 3.09\% per year; HR $0.69,95 \%$ CI $0.60-0.80, P<0.001)$. Major and minor bleeding complications as defined by Global Use of Strategies to Open Occluded Arteries (GUSTO) or Thrombolysis in Myocardial Infarction (TIMI) criteria were also less frequent in the apixaban group $(P<0.001)$ [12]. Importantly, there was no significant difference in the incidence of gastrointestinal hemorrhage between groups $(0.76 \% \mathrm{vs} .0 .86 \%$ per year: HR $0.89,95 \%$ CI $0.70-1.15, P=0.37$ ). Along with the reduction in the hemorrhagic strokes, there was also a reduction in all intracranial hemorrhages (composite of intracerebral hemorrhage, subarachnoid hemorrhage and subdural hemorrhage) in patients receiving apixaban ( $0.33 \%$ vs. $0.80 \%$; HR $0.42,95 \%$ CI $0.30-0.58, P<0.001)$. Lastly, patients randomized to apixaban had a lower mortality $(3.52 \%$ vs. $3.94 \%$ per year, $P=0.047)$ and more frequently a favorable net clinical outcome, defined as composite of stroke, systemic embolism, major bleeding and death from any cause $(6.13 \%$ vs. $7.20 \%$ per year; HR 0.85, 95\% CI 0.78-0.92, $P<0.001)$.

\section{Apixaban vs. Aspirin}

Aspirin use is recommended for primary and secondary stroke prevention in patients with atrial fibrillation, who are not suitable for treatment with vitamin $\mathrm{K}$ antagonists due to higher risk for bleeding complications [13]. The Apixaban vs. Acetylsalicylic Acid to Prevent Stroke in Atrial Fibrillation Patients (AVERROES) trial [14] was a double blind, multicenter trial, designed to determine the efficacy and safety of apixaban $v s$. aspirin in patients with atrial fibrillation and at least one more vascular risk factor for stroke who were not suitable to receive vitamin $\mathrm{K}$ antagonists. This trial included 5599 patients.

Apixaban was more effective in preventing strokes (ischemic or hemorrhagic) and systemic embolism as compared to aspirin ( $1.6 \%$ vs. $3.7 \%$ per year, HR with apixaban $0.45,95 \%$ CI 0.32 to $0.62 ; P<0.001)$. On further analysis, apixaban was associated with a greater reduction in the annual incidence in ischemic strokes $(1.1 \% v s .3 .0 \%$ per year, HR with apixaban $0.37 ; 95 \% \mathrm{CI}, 0.25$ to $0.55 ; P<0.001)$ and there was a similar incidence in intracranial hemorrhages $(0.4 \%$ vs. $0.4 \%$ per year, HR with apixaban $0.85,95 \%$ CI $0.38-1.90 ; P=0.69)$ as well as all hemorrhagic strokes between groups $(0.2 \%$ vs. $0.3 \%$ per year, HR $0.67,95 \%$ CI $0.24-1.88 ; P=0.45)$. Finally, apixaban was associated with an increase in the annual incidence of minor bleeding complications $(6.3 \%$ vs. $5.0 \%$ per year; $P=0.05)$ but no difference in the annual incidence of major bleeding $(1.4 \% \mathrm{vs} .1 .2 \%$ per year; $P=0.57)$ and fatal bleeding complications $(0.1 \% \mathrm{vs}$. $0.2 \%$ per year; $P=0.53$ ) as compared to aspirin. Results were overall similar in an on-treatment analysis restricted to events occurring while patients were taking the study drug. In this analysis apixaban was associated with a greater annual incidence of major bleeding events $(1.4 \%$ vs. $0.9 \%$ per year; $P=0.07)$.

The results of the AVERROES study have significant implications for a substantial proportion of patients who are 
not considered candidates for anticoagulation therapy and are at risk of stroke $[15,16]$.

\section{Rivaroxaban}

Rivaroxaban has a high oral bioavailability and is well absorbed. It has a rapid onset of action within about 2-4 hours of administration and a half-life ranging from 7-13 hours (longer half-lives are observed in elderly individuals). Similar to apixaban it has a dual mode of excretion with approximately one third being excreted unchanged through kidneys and two thirds metabolized by the liver to inactive metabolites [17].

Rivaroxaban Once Daily Oral Direct Factor Xa Inhibition Compared with Vitamin K Antagonism for Prevention of Stroke and Embolism Trial in Atrial Fibrillation (ROCKET AF) was a non-inferiority, double blind trial comparing rivaroxaban $20 \mathrm{mg}$ once a day (or $15 \mathrm{mg}$ once a day for patients with creatinine clearance of 30-49 ml/minute) to dose adjusted warfarin in patients with non-valvular atrial fibrillation with moderate risk for stroke (defined as a $\mathrm{CHADS}_{2}$ score of $\geq 2$ ) [18]. This trial enrolled 14264 patients, median duration of treatment exposure was 590 days and median follow up duration was 707 days.

In the primary or per-protocol analysis, rivaroxaban was non-inferior to warfarin in preventing stroke (composite of ischemic or hemorrhagic stroke) or systemic embolism (1.7\% vs. $2.2 \%$ per year; HR $0.79,95 \%$ CI $0.66-0.96, P<0.001)$. The incidence of myocardial infarction $(0.9 \%$ vs. $1.1 \%$ per year; HR $0.81,95 \%$ CI $0.63-1.06, P=0.12)$ and death $(1.9 \%$ vs. $2.2 \%$ per year; HR $0.85,95 \%$ CI $0.70-1.02, P=0.07)$ was similar between groups.

There was no difference in major and non-major clinically relevant bleeding complications between the groups (14.9\% vs. $14.5 \%$ per year; HR $1.03,95 \%$ CI $0.96-1.11, P=0.44)$. However, intracranial hemorrhages $(0.5 \%$ vs. $0.7 \%$ per year; HR $0.67,95 \%$ CI $0.47-0.93, P=0.02)$ and fatal bleeding complications $(0.2 \%$ vs. $0.5 \%$ per year; HR $0.50,95 \%$ CI $0.31-0.79, P=0.003)$ were reduced in the rivaroxaban arm. Major gastrointestinal bleeding was more common in the rivaroxaban arm $(3.2 \%$ vs. $2.2 \%$ per year, $P<0.001)$.

\section{Edoxaban}

Edoxaban also has relatively high oral bioavailability with quick absorption and peak plasma concentration within 1.5 hours of administration. It has a half-life of approximately 10-14 hours [19].

The Effective Anticoagulation with Factor Xa Next Generation in Atrial Fibrillation - Thrombolysis in Myocardial Infarction 48 (ENGAGE AF TIMI 48) was a double blind, randomized, double-dummy, non-inferiority trial comparing two doses $(60 \mathrm{mg}$ once a day in high dose group and $30 \mathrm{mg}$ once a day in low dose group, the dose was halved in either group if any of the following characteristics was present: creatinine clearance of 30 to $50 \mathrm{ml} /$ minute, body weight $\leq 60$ kilograms, concomitant use of verapamil or quinidine) of edoxaban to dose adjusted warfarin (goal INR 2.0-3.0) in patients with atrial fibrillation with a moderate to high risk for stroke (defined as a $\mathrm{CHADS}_{2}$ score of $\geq 2$ ) [20]. This trial enrolled 21105 patients with median follow up duration of 2.8 years.

In the modified intention to treat analysis (population in the treatment period), there were fewer strokes or systemic embolism in the high dose edoxaban group as compared to warfarin group $(1.18 \%$ vs. $1.50 \%$ per year; $\mathrm{HR} 0.79,95 \% \mathrm{CI}$ $0.63-0.99, P<0.001$ for non-inferiority). In the intention to treat analysis (population in the overall study period), there was a non-significant trend in favor of high dose edoxaban in preventing stroke (composite of ischemic or hemorrhagic stroke) or systemic embolisms ( $1.57 \%$ vs. $1.80 \%$ per year; HR $0.87,95 \%$ CI $0.73-1.04, P=0.08)$. The overall stroke incidence was similar between groups (1.49\% per year in the high dose edoxaban $v s .1 .69 \%$ per year in warfarin group; HR $0.88,95 \%$ CI $0.75-1.03, P=0.11$ ). However, there was a there was a $46 \%$ relative reduction in hemorrhagic strokes in the high dose edoxaban group $(0.26 \%$ vs. $0.47 \%$ per year; HR $0.54,95 \%$ CI $0.38-0.77, P<0.001)$ while the incidence of ischemic strokes was similar between treatment arms (1.25\% per year in either group; HR 1.00, 95\% CI 0.83-1.19, $P=0.97)$. A significant $20 \%$ relative reduction in non-disabling or non-fatal strokes was noted in the high dose edoxaban group as compared to warfarin, but the number of fatal and disabling strokes was similar between arms. The composite major adverse cardiac events, defined as myocardial infarction, stroke, systemic embolism or death from cardiovascular cause, were less frequent in the high dose edoxaban group (4.41\% vs. 4.98\% per year; HR 0.88, 95\% CI 0.81-0.97, $P=0.01)$. The incidence of myocardial infarction remained same in both the groups $(0.70 \% v s .0 .75 \%$ per year; HR 0.94 , 95\% CI 0.74-1.19, $P=0.60)$.

There was a significant reduction in bleeding complications in the high dose edoxaban group including major bleeding (2.75\% vs. 3.43\% per year; HR 0.80, 95\% CI $0.71-0.91, P<0.001)$, intracranial hemorrhages $(0.39 \% v s .0 .85 \%$ per year; HR $0.47,95 \%$ CI $0.34-0.63, P<0.001)$, and fatal intracranial hemorrhages $(0.15 \%$ vs. $0.27 \%$ per year; HR 
0.58, 95\% CI 0.35-0.95, $P=0.03)$. Conversely, a higher incidence of gastrointestinal bleeding was observed in the high dose edoxaban group $(1.51 \%$ vs. $1.23 \%$ per year; HR $1.23,95 \%$ CI $1.02-1.50, P=0.03)$. Other hemorrhagic complications including fatal hemorrhages, life threatening hemorrhages, minor bleeding, clinically relevant non major bleeding and any overt bleeding complications were significantly reduced in the high dose edoxaban group.

\section{Summary of Doac Trials}

Meta-analysis of the four above summarized DOAC-trials (RE-LY, ROCKET AF, ARISTOTLE, ENGAGE AFTIMI) included as a group vs. dose adjusted warfarin for preventing stroke indicated that high dose DOACs significantly reduce the incidence of all stroke (ischemic or hemorrhagic stroke) and systemic embolism by $19 \%$ as compared to Warfarin (RR $0.81,95 \%$ CI $0.73-0.91, P<0.0001$ ) [21]. This benefit was mainly driven by a substantial $51 \%$ reduction in hemorrhagic strokes (RR 0.49, 95\% CI 0.38-0.64, $P<0.0001)$ [21].

Further, compared to warfarin, treatment with DOACs was associated with a non-significant $18 \%$-reduction in ischemic strokes (RR $0.92,95 \%$ CI $0.83-1.02, P=0.1)$ and a similar incidence of myocardial infarction (RR $0.97,95 \%$ CI $0.78-1.20, P=0.77$ ). DOACs use was associated with a reduction in all-cause mortality (RR $0.90,95 \%$ CI $0.85-0.95$, $P=0.0003$ ). Importantly, DOAC-treatment was associated with a significant reduction in incident intracranial hemorrhages (combination of hemorrhagic stroke, subarachnoid hemorrhage, subdural hemorrhage and epidural hemorrhage) (RR 0.48, 95\% CI 0.39-0.59, $P<0.0001)$ but increased risk for gastrointestinal bleeding (RR $1.25,95 \%$ CI 1.01-1.55, $P=0.043$ ) [21]. Overall, there was no significant difference in the incidence of stroke or systemic embolism the following subgroups as stratified by: age $<75 \mathrm{vs} . \geq 75$ years; sex; presence of diabetes; history of stroke or transient ischemic attack; degree of renal impairment; $\mathrm{CHADS}_{2}$-score; time in therapeutic range; vitamin $\mathrm{K}$ antagonist status at study entrance [21].

Similarly, there was no significant difference in the incidence of major bleeding complications except for the time in therapeutic range $(<66 \% v s . \geq 66 \%)$. Specifically, there was a greater relative reduction in major bleeding complications with DOAC therapy, when the center-based time in the therapeutic range was $<66 \%$ (RR $0.69 v s .0 .93, P=0.022$ ), suggesting that efficacy and safety of DOACs is independent of how well warfarin therapy is managed and may be particularly useful for patients whose INR is difficult to maintain [21].

When the meta-analysis was restricted to factor Xa inhibitors, the overall results were unchanged. Additional sensitivity analyses indicated that low dose DOAC treatment (which is not approved by the FDA in the United States) provided similar efficacy in preventing stroke and systemic embolism as compared to warfarin. Though ischemic strokes were more frequent in this group as compared to warfarin, this effect was offset by a reduction in the incidence of hemorrhagic strokes. Similar to higher dose DOACs, low dose DOACs were associated with a reduction in all-cause mortality as well as similar incidence of gastrointestinal hemorrhages.

\section{Advantages of Doac Over Warfarin Use}

DOACs use does not require dietary restrictions, which is an advantage of warfarin - which may exert unstable anticoagulation effects depending on the amount of consumed vitamin $\mathrm{K}$ and thus result in increased risk for thromboembolism or hemorrhagic complications in the case of subtherapeutic and supratherapeutic levels, respectively [22].

Furthermore, their fewer drug-drug interactions render DOACs a more suitable option for many patients with atrial fibrillation that have multiple comorbid conditions and require taking multiple medications [23].

In addition to having fewer dietary and drug-drug interactions, DOACs are associated with a relatively steady state plasma concentration, leading to lesser fluctuation in the anticoagulation effect that obviates the need for frequent and regular laboratory monitoring and may contribute to observed fewer bleeding complications as compared to warfarin [21].

DOACs have quick absorption, shorter peak time leading to quicker onset of steady state anticoagulation effect as compared to warfarin, which requires bridging with other form of antiplatelet or anticoagulants by the time optimal anticoagulation effect is achieved. Conversely, after discontinuation DOACs are associated with relatively quick offset of anticoagulant effect due to their shorter half-life and absent need for resynthesis of the vitamin $\mathrm{K}$ dependent coagulation factors by the liver. 


\section{DISADVANTAGES OVER WARFARIN USE}

\section{DOAC Reversal}

In contrast to warfarin, which can be relatively quickly reversed with recombinant Factor VIIa (rFVIIa) and prothrombin complex concentrate (PCC) [24], there is presently no effective and established treatment strategy to reverse the action of DOACs, which is hampered as a result of their competitive inhibition of thrombin or activated factor X (as opposed to clotting factor deficiency).

Nevertheless, experimental data in a mouse model of ICH associated with dabigatran, PCC, but not rFVIIa or fresh frozen plasma (FFP), prevented intracerebral hematoma expansion [25]. Conversely, in a rabbit model, rFVIIa and PCC partially improved laboratory parameters but failed to reverse rivaroxaban associated bleeding [26]. However, rivaroxaban-related, but not dabigatran-related, coagulopathy could be reversed with PCC in a randomized, double blind placebo controlled trial in healthy volunteers [26]. Clinically, emergent hemodialysis could expedite clearance of DOACs, which are renally eliminated to a substantial degree (see above) [16]. Indeed, in patients with end-stage renal disease, $62-68 \%$ of dabigatran was removed with hemodialysis of 4 hours duration [26]. However, this approach may be of limited practical utility and efficacy of this approach remains to be shown. Also, hemodialysis may not effectively removing rivaroxaban and apixaban, which are largely protein bound.

\section{DOAC Antidotes in Development}

Several specific antidotes for DOAC-reversal are presently in development [27]. Idarucizumab, which is a humanized antibody fragment against dabigatran that has a 350 times higher affinity to thrombin than dabigatran. It has been shown to successfully reverse the effect of dabigatran by examining clotting time in the rat [28]. The RE-VERSal Effects of Idarucizumab on Active Dabigatran (REVERSE-AD) trial is an ongoing international study, to investigate its role as a potential antidote in the clinical setting in patients taking dabigatran [NCT02104947].

The recombinant antidote protein PRT064445 is a modified form of factor Xa, which acts by reducing plasma anti factor Xa activity and the non-protein bound fraction of factor Xa inhibitors. In rabbits treated with rivaroxaban, PRT064445 restored hemostasis after liver laceration, showing promise as a potential antidote to reverse factor Xa inhibitor associated coagulopathy [29].

PER977 is a small, synthetic, water soluble cationic molecule, designed to bind specifically to unfractionated heparin and low molecular weight heparin. Similarly, it can also bind to oral factor Xa inhibitors as well as direct thrombin inhibitors. In 80 healthy volunteers, by checking whole blood clotting time, PER977 was able to restore baseline hemostasis from anticoagulated state (achieved with edoxaban) within 10 to 30 minutes of administration and effect was sustained for 24 hours [30].

While these agents are promising in reversing the coagulopathy related to DOACs their clinical safety and efficacy remains to be established.

\section{Anticoagulation Efficacy}

In contrast to warfarin, routine laboratory testing with activated partial thromboplastin time (aPTT) and International normalized ratio (INR) are insensitive to accurately determine the relative intensity of DOAC [31]. Though, dilute thrombin time (dTT), ecarin clotting time (ECT) and ecarin chromogenic assay (ECA) in case of dabigatran and chromogenic anti Xa assay in case of factor Xa inhibitors could be used to better quantify the anticoagulation effect, they are not widely available, known to have inter-lot variability, and may be challenging to perform in a timely fashion [31].

\section{Thrombotic Events After Sudden Discontinuation of DOACs}

In light of their relatively short half-life, anticoagulant effect of DOACs is lost within 48-72 hours from the last dose leaving patients at potential risk for thromboembolic complications. This is highlighted by the observation of an increased incidence in strokes and systemic embolic events after discontinuation of rivaroxaban as well as apixaban while transitioning to open-label warfarin at the end of the study $[32,33]$. These observations led to a boxed warning for all DOACs by the US Food and Drug Administration to avoid sudden discontinuation except in the setting of hemorrhagic complications or completion of therapy [33]. It is important to note that the excess in thrombotic complications did not appear to result from a "rebound" hypercoagulability but was rather related to the known 
challenges with initiating warfarin therapy after clinical trial completion $[32,33]$. Accordingly, it seems prudent to consider adequate alternative anticoagulation coverage during planned interruptions. Nevertheless, it is presently unknown whether bridging anticoagulation will produce a net clinical benefit and which approach to choose [32]. Further clinical research and post marketing data analysis is needed to help clarify this critical issue. Until such data becomes available it is important to counsel patients, minimize interruptions in therapy, and pay close attention to the INR when transitioning to warfarin.

\section{Comparison Among DOACs}

There is no trial to date directly comparing DOACs. All DOACs provide equal or greater protection against allcause strokes as compared to dose adjusted warfarin, a benefit mainly driven by the reduction in hemorrhagic stroke [8, $11,18,20,21]$ With reference to preventing ischemic stroke (Table 1), dabigatran appears to be more promising as compared to warfarin in preventing ischemic strokes [8] but all other DOACs provide similar benefit in preventing ischemic strokes in comparison to warfarin. All DOACs appear to have a more favorable safety profile compared to warfarin in reducing bleeding complications including intracranial hemorrhages though they are associated with an increased risk for gastrointestinal hemorrhages $[14,18,20]$ except for apixaban, which was shown to have similar risk of gastrointestinal hemorrhage as warfarin in ARISTOTLE trial [11].

Table 1. Comparison of NOACs with Warfarin (Annual incidence rate).

\begin{tabular}{|c|c|c|c|c|c|c|c|c|c|c|c|c|}
\hline \multirow{2}{*}{$\begin{array}{c}\text { Outcome } \\
\text { measures in } \\
\text { the clinical } \\
\text { Trials }\end{array}$} & \multicolumn{3}{|c|}{ RE-LY } & \multicolumn{3}{|c|}{ Rocket AF } & \multicolumn{3}{|c|}{ Aristotle } & \multicolumn{3}{|c|}{ Engage TIMI AF } \\
\hline & \begin{tabular}{|c|} 
Dabigatran \\
$(150 \mathrm{mg}$ \\
only $)$
\end{tabular} & Warfarin & $\begin{array}{c}\text { RR }(95 \% \\
\text { CI) }\end{array}$ & Rivaroxaban & Warfarin & $\begin{array}{c}\text { HR }(95 \% \\
\text { CI })\end{array}$ & Apixaban & Warfarin & $\begin{array}{c}\text { HR }(95 \% \\
\text { CI) }\end{array}$ & \begin{tabular}{|c|} 
Edoxaban \\
$(60 \mathrm{mg}$ \\
only)
\end{tabular} & Warfarin & $\begin{array}{c}\text { HR }(95 \% \\
\text { CI) }\end{array}$ \\
\hline $\begin{array}{l}\text { Composite of } \\
\text { strokes and } \\
\text { systemic } \\
\text { embolisms }\end{array}$ & $1.11 \%$ & $1.69 \%$ & $\begin{array}{c}0.66 \\
(0.53-0.82)\end{array}$ & $1.70 \%$ & $2.20 \%$ & $\begin{array}{c}0.79 \\
(0.66-0.96)\end{array}$ & $1.27 \%$ & $1.60 \%$ & \begin{tabular}{|c|}
0.79 \\
$(0.66-0.95)$
\end{tabular} & $1.18 \%$ & $1.50 \%$ & $\begin{array}{c}0.87 \\
(0.73-1.04)\end{array}$ \\
\hline $\begin{array}{l}\text { Ischemic } \\
\text { strokes }\end{array}$ & $0.92 \%$ & $1.20 \%$ & \begin{tabular}{|c|}
0.76 \\
$(0.60-0.98)$ \\
\end{tabular} & $1.34 \%$ & $1.42 \%$ & $\begin{array}{c}0.94 \\
(0.75-1.17) \\
\end{array}$ & $0.97 \%$ & $1.05 \%$ & \begin{tabular}{|c|}
0.92 \\
$(0.74-1.13)$ \\
\end{tabular} & $1.25 \%$ & $1.25 \%$ & $\begin{array}{c}1.00 \\
(0.83-1.19)\end{array}$ \\
\hline $\begin{array}{l}\text { Hemorrhagic } \\
\text { strokes }\end{array}$ & $0.10 \%$ & $0.38 \%$ & \begin{tabular}{|c|}
0.26 \\
$(0.14-0.49)$
\end{tabular} & $0.26 \%$ & $0.44 \%$ & $\begin{array}{c}0.59 \\
(0.37-0.93)\end{array}$ & $0.24 \%$ & $0.47 \%$ & $\begin{array}{c}0.51 \\
(0.35-0.75)\end{array}$ & $0.26 \%$ & $0.47 \%$ & $\begin{array}{c}0.54 \\
(0.38-0.77)\end{array}$ \\
\hline $\begin{array}{l}\text { Intracranial } \\
\text { hemorrhages }\end{array}$ & $0.30 \%$ & $0.74 \%$ & $\begin{array}{c}0.40 \\
(0.27-0.60) \\
\end{array}$ & $0.50 \%$ & $0.70 \%$ & $\begin{array}{c}0.67 \\
(0.47-0.93) \\
\end{array}$ & $0.33 \%$ & $0.80 \%$ & $\begin{array}{c}0.42 \\
(0.30-0.58) \\
\end{array}$ & $0.39 \%$ & $0.85 \%$ & $\begin{array}{c}0.47 \\
(0.34-0.63)\end{array}$ \\
\hline $\begin{array}{c}\text { Gastrointestinal } \\
\text { hemorrhages }\end{array}$ & $1.51 \%$ & $1.02 \%$ & $\begin{array}{c}1.50 \\
(1.19-1.89) \\
\end{array}$ & $3.20 \%$ & $2.20 \%$ & $--^{*}$ & $0.76 \%$ & $0.86 \%$ & $\begin{array}{c}0.89 \\
(0.70-1.15) \\
\end{array}$ & $1.51 \%$ & $1.23 \%$ & $\begin{array}{c}1.23 \\
(1.02-1.50) \\
\end{array}$ \\
\hline $\begin{array}{l}\text { All-cause } \\
\text { mortality }\end{array}$ & $3.64 \%$ & $4.13 \%$ & $\begin{array}{c}0.88 \\
(0.77-1.00)\end{array}$ & $4.50 \%$ & $4.90 \%$ & $\begin{array}{c}0.92 \\
(0.82-1.03)\end{array}$ & $3.52 \%$ & $3.94 \%$ & $\begin{array}{c}0.89 \\
(0.80-0.99)\end{array}$ & $3.99 \%$ & $4.35 \%$ & $\begin{array}{c}0.92 \\
(0.83-1.01)\end{array}$ \\
\hline
\end{tabular}

$\mathrm{CI}=$ confidence interval; $\mathrm{HR}=$ hazard ratio; $\mathrm{RR}=$ relative risk. ${ }^{*}$ not provided.

\section{REMAINING AREAS FOR CLARIFICATION}

\section{DOAC Use in the Elderly}

DOACs ability of preventing stroke or systemic embolism as compared to warfarin was not dependent of patient's age but at the same time, greater reduction in the major bleeding complications noted in the younger (age $<75$ years) patients as compared to elderly patients $[16,21]$. For example, the risk of major extracranial hemorrhage increased with dabigatran compared with warfarin with increasing age; nevertheless, the risk of intracranial bleeding remained lower in the dabigatran arms [34]. Similarly, rivaroxaban is associated with an increased risk for non-major clinically relevant bleeding in patients aged $\geq 75$ years compared with warfarin [35]. Though, DOACs reduce all-cause stroke and systemic embolism including in the elderly and do not appear to increase intracranial hemorrhages [16], further study of their efficacy and safety particularly with respect to extracranial hemorrhages in the very old require further study.

\section{Patients at Low Risk for Ischemic Stroke}

The majority of patients included in the major trials comparing DOACs to warfarin included patients with moderate to high risk for stroke $\left(\mathrm{CHADS}_{2}\right.$ score $\left.>1\right)$. Though meta analysis of these trials did not show a significant difference between risk groups, DOACs superiority over warfarin appeared to be driven by inclusion of patients at high stroke risk $\left(\mathrm{CHADS}_{2} \geq 3\right)[21]$. Accordingly, warfarin remains a viable alternative to DOACs particularly among low risk subjects. 


\section{Patients with Embolic Stroke of Undetermined Source}

On the basis of the apparent low hemorrhagic risk $[8,11,18,20,21]$, the efficacy and safety to DOACs for secondary stroke prevention in patients with Embolic Stroke of Undetermined Source (ESUS) is being evaluated by two ongoing clinical trials: RE-SPECT ESUS compares dabigatran to aspirin [NCT02239120] and NAVIGATE ESUS compares rivaroxaban to aspirin [NCT02313909].

\section{Combination of DOACs with Antiplatelets}

As per the American Heart Association Guidelines combination of anticoagulants and antiplatelet therapy is not recommended for the prevention of stroke and transient ischemic attack but may be reasonable in patients with clinically apparent coronary artery disease particularly acute coronary syndrome (Class IIb and level C evidence) [13]. In the subgroup analysis of RE-LY, concomitant use of antiplatelets was associated with increased risk for major bleeding complications without additional benefits of preventing ischemic complications [8]. In time dependent analysis, concomitant use of a single antiplatelet agent and dabigatran (150 mg twice a day) was associated with increased risk for major bleeding complications (HR 1.60, 95\% CI 1.42-1.82) and concomitant use of double antiplatelet agents further increased that risk (HR 2.31, 95\% CI 1.79-2.98) [36]. Accordingly, combination therapy of DOACs with antiplatelets should generally be avoided.

\section{DOACs Management in the Perioperative Period}

There are no specific guidelines regarding DOAC use in the perioperative period available at this time. As per the manufacture guidelines, Dabigatran should be discontinued without bridging ranging from 2 to 5 days before the procedure depending upon renal function and risk of bleeding from procedure [37]. Rivaroxaban is recommended to be discontinued for 24 hours before the invasive procedure and 36-48 hours before the surgery with high bleeding risk and any surgery involving the central nervous system [38]. Apixaban should be discontinued for 24 hours before planned surgery with low bleeding risk and for at least 48 hours before the moderate to high bleeding risk surgery or invasive procedure [39].

\section{Thrombolysis and Endovascular Therapy Decision Making in Acute Ischemic Stroke in Patients Treated with DOACs}

The increasing use of DOACs creates a challenge to acute ischemic stroke therapy with intravenous tissue-type plasminogen activator (rtPA) in otherwise eligible patients [40, 41]. As previously discussed in this article, routine and emergently available laboratory testing that reliably inform on anticoagulant effect in patients taking DOACs are not yet available [31, 42]. While timing of the last dose of administration before hospital presentation could potentially help determine the relative anticoagulant effect of DOACs it remains unknown whether this approach is safe. Accordingly, routine administration of systemic rtPA in patients on DOACs is not recommended and great caution should be applied when considering thrombolysis even if available conventional laboratory essays for anticoagulant activity (such as the aPTT and INR) appear normal and time from last dose has been several hours [42]. Similarly, for patients presenting with an acute large arterial occlusion endovascular stroke therapy may be considered [43, 44]; however, it should be noted that none of the recent large randomized trials informed on safety and efficacy of endovascular stroke therapy in patients on DOACs [45 - 49]. Thus, further clinical research is needed to clarify these important issues and therapeutic decisions should be carefully weighted in each patient.

\section{CONCLUSION}

DOACs represent an appealing alternative to warfarin for the primary and secondary stroke prevention patients with atrial fibrillation. DOACs as a group are more effective than dose adjusted warfarin for prevention of all-cause stroke (including both ischemic and hemorrhagic stroke), and they have an overall more favorable safety profile. Nevertheless, increased risk for gastrointestinal bleeding (with the exception of apixaban) and risk for thrombotic complication with sudden discontinuation as well as inability to accurately assess anticoagulant efficacy and reverse the anticoagulant effect require consideration prior to therapy initiation and pose a challenge for decision making in acute stroke therapy.

\section{CONFLICT OF INTEREST}

The authors confirm that this article content has no conflicts of interest. 


\section{ACKNOWLEDGEMENTS}

Declared none.

\section{REFERENCES}

[1] Hart RG, Pearce LA, Aguilar MI. Meta-analysis: antithrombotic therapy to prevent stroke in patients who have nonvalvular atrial fibrillation. Ann Intern Med 2007; 146(12): 857-67. [http://dx.doi.org/10.7326/0003-4819-146-12-200706190-00007] [PMID: 17577005]

[2] Go AS, Hylek EM, Borowsky LH, Phillips KA, Selby JV, Singer DE. Warfarin use among ambulatory patients with nonvalvular atrial fibrillation: the anticoagulation and risk factors in atrial fibrillation (ATRIA) study. Ann Intern Med 1999; 131(12): 927-34. [http://dx.doi.org/10.7326/0003-4819-131-12-199912210-00004] [PMID: 10610643]

[3] Granger CB, Armaganijan LV. Newer oral anticoagulants should be used as first-line agents to prevent thromboembolism in patients with atrial fibrillation and risk factors for stroke or thromboembolism. Circulation 2012; 125(1): 159-64. [http://dx.doi.org/10.1161/CIRCULATIONAHA.111.031146] [PMID: 22215890]

[4] The Stroke Prevention in Atrial Fibrillation Investigators. Bleeding during antithrombotic therapy in patients with atrial fibrillation. Arch Intern Med 1996; 156(4): 409-16. [http://dx.doi.org/10.1001/archinte.1996.00440040081009] [PMID: 8607726]

[5] van der Meer FJ, Rosendaal FR, Vandenbroucke JP, Briët E. Bleeding complications in oral anticoagulant therapy. An analysis of risk factors Arch Intern Med 1993; 153(13): 1557-62. [http://dx.doi.org/10.1001/archinte.1993.00410130073007] [PMID: 8323419]

[6] Landefeld CS, Goldman L. Major bleeding in outpatients treated with warfarin: incidence and prediction by factors known at the start of outpatient therapy. Am J Med 1989; 87(2): 144-52. [http://dx.doi.org/10.1016/S0002-9343(89)80689-8] [PMID: 2787958]

[7] Ganetsky M, Babu KM, Salhanick SD, Brown RS, Boyer EW. Dabigatran: review of pharmacology and management of bleeding complications of this novel oral anticoagulant. J Med Toxicol 2011; 7(4): 281-7. [http://dx.doi.org/10.1007/s13181-011-0178-y] [PMID: 21887485]

[8] Connolly SJ, Ezekowitz MD, Yusuf S, et al. RE-LY Steering Committee and Investigators. Dabigatran vs. warfarin in patients with atrial fibrillation. N Engl J Med 2009; 361(12): 1139-51. [http://dx.doi.org/10.1056/NEJMoa0905561] [PMID: 19717844]

[9] Frost C, Wang J, Nepal S, et al. Apixaban, an oral, direct factor Xa inhibitor: single dose safety, pharmacokinetics, pharmacodynamics and food effect in healthy subjects. Br J Clin Pharmacol 2013; 75(2): 476-87. [http://dx.doi.org/10.1111/j.1365-2125.2012.04369.x] [PMID: 22759198]

[10] Frost C, Nepal S, Wang J, et al. Safety, pharmacokinetics and pharmacodynamics of multiple oral doses of apixaban, a factor Xa inhibitor, in healthy subjects. Br J Clin Pharmacol 2013; 76(5): 776-86. [http://dx.doi.org/10.1111/bcp.12106] [PMID: 23451769]

[11] Granger CB, Alexander JH, McMurray JJ, et al. Apixaban vs. warfarin in patients with atrial fibrillation. N Engl J Med 2011; 365(11): 981-92.

[http://dx.doi.org/10.1056/NEJMoa1 107039] [PMID: 21870978]

[12] Mehran R, Rao SV, Bhatt DL, et al. Standardized bleeding definitions for cardiovascular clinical trials: a consensus report from the Bleeding Academic Research Consortium. Circulation 2011; 123(23): 2736-47. [http://dx.doi.org/10.1161/CIRCULATIONAHA.110.009449] [PMID: 21670242]

[13] Kernan WN, Ovbiagele B, Black HR, et al. American Heart Association Stroke Council, Council on Cardiovascular and Stroke Nursing, Council on Clinical Cardiology, and Council on Peripheral Vascular Disease. Guidelines for the prevention of stroke in patients with stroke and transient ischemic attack: a guideline for healthcare professionals from the American Heart Association/American Stroke Association. Stroke 2014; 45(7): 2160-236. [http://dx.doi.org/10.1161/STR.0000000000000024] [PMID: 24788967]

[14] Connolly SJ, Eikelboom J, Joyner C, et al. AVERROES Steering Committee and Investigators. Apixaban in patients with atrial fibrillation. N Engl J Med 2011; 364(9): 806-17.

[http://dx.doi.org/10.1056/NEJMoa1007432] [PMID: 21309657]

[15] Birman-Deych E, Radford MJ, Nilasena DS, Gage BF. Use and effectiveness of warfarin in Medicare beneficiaries with atrial fibrillation. Stroke 2006; 37(4): 1070-4. [http://dx.doi.org/10.1161/01.STR.0000208294.46968.a4] [PMID: 16528001]

[16] $\mathrm{Ng} \mathrm{KH}$, Hart RG, Eikelboom JW. Anticoagulation in patients aged $>/=75$ years with atrial fibrillation: role of novel oral anticoagulants. Cardiol Ther 2013; 2(2): 135-49.

[http://dx.doi.org/10.1007/s40119-013-0019-y] [PMID: 25135392]

[17] Kreutz R. Pharmacodynamic and pharmacokinetic basics of rivaroxaban. Fundam Clin Pharmacol 2012; 26(1): 27-32. [http://dx.doi.org/10.1111/j.1472-8206.2011.00981.x] [PMID: 21848931]

[18] Patel MR, Mahaffey KW, Garg J, et al. ROCKET AF Investigators.. Rivaroxaban vs. warfarin in nonvalvular atrial fibrillation. N Engl J Med 
2011; 365(10): 883-91.

[http://dx.doi.org/10.1056/NEJMoa1009638] [PMID: 21830957]

[19] Lip GY, Agnelli G. Edoxaban: a focused review of its clinical pharmacology. Eur Heart J 2014; 35(28): 1844-55. [http://dx.doi.org/10.1093/eurheartj/ehu181] [PMID: 24810388]

[20] Giugliano RP, Ruff CT, Braunwald E, et al. ENGAGE AF-TIMI 48 Investigators.. Edoxaban vs. warfarin in patients with atrial fibrillation. N Engl J Med 2013; 369(22): 2093-104. [http://dx.doi.org/10.1056/NEJMoa1310907] [PMID: 24251359]

[21] Ruff CT, Giugliano RP, Braunwald E, et al. Comparison of the efficacy and safety of new oral anticoagulants with warfarin in patients with atrial fibrillation: a meta-analysis of randomised trials. Lancet 2014; 383(9921): 955-62. [http://dx.doi.org/10.1016/S0140-6736(13)62343-0] [PMID: 24315724]

[22] Couris R, Tataronis G, McCloskey W, et al. Dietary vitamin K variability affects International Normalized Ratio (INR) coagulation indices Int J Vitam Nutr Res 2006; 76(2): 65-74.

[http://dx.doi.org/10.1024/0300-9831.76.2.65] [PMID: 16941417]

[23] Bauer KA. Pros and cons of new oral anticoagulants. Am Soc Hematol Educ Prog 2013; 2013: 70-464. [http://dx.doi.org/10.1182/asheducation-2013.1.464]

[24] Garcia DA, Crowther MA. Reversal of warfarin: case-based practice recommendations. Circulation 2012; $125(23): 2944-7$. [http://dx.doi.org/10.1161/CIRCULATIONAHA.111.081489] [PMID: 22689931]

[25] Zhou W, Schwarting S, Illanes S, et al. Hemostatic therapy in experimental intracerebral hemorrhage associated with the direct thrombin inhibitor dabigatran. Stroke 2011; 42(12): 3594-9.

[http://dx.doi.org/10.1161/STROKEAHA.111.624650] [PMID: 21998060]

[26] Eerenberg ES, Kamphuisen PW, Sijpkens MK, Meijers JC, Buller HR, Levi M. Reversal of rivaroxaban and dabigatran by prothrombin complex concentrate: a randomized, placebo-controlled, crossover study in healthy subjects. Circulation 2011; 124(14): 1573-9. [http://dx.doi.org/10.1161/CIRCULATIONAHA.111.029017] [PMID: 21900088]

[27] Costin J, Ansell J, Laulicht B, Bakhru S, Steiner S. Reversal agents in development for the new oral anticoagulants. Postgrad Med 2014; 126(7): 19-24. [http://dx.doi.org/10.3810/pgm.2014.11.2829] [PMID: 25387210]

[28] Schiele F, van Ryn J, Canada K, et al. A specific antidote for dabigatran: functional and structural characterization. Blood 2013; 121(18): 3554-62.

[http://dx.doi.org/10.1182/blood-2012-11-468207] [PMID: 23476049]

[29] Lu G, DeGuzman FR, Hollenbach SJ, et al. A specific antidote for reversal of anticoagulation by direct and indirect inhibitors of coagulation factor Xa. Nat Med 2013; 19(4): 446-51. [http://dx.doi.org/10.1038/nm.3102] [PMID: 23455714]

[30] Ansell JE, Bakhru SH, Laulicht BE, et al. Use of PER977 to reverse the anticoagulant effect of edoxaban. N Engl J Med 2014; 371(22): 2141-2.

[http://dx.doi.org/10.1056/NEJMc1411800] [PMID: 25371966]

[31] Douxfils J, Tamigniau A, Chatelain B, Goffinet C, Dogné JM, Mullier F. Measurement of non-VKA oral anticoagulants vs. classic ones: the appropriate use of hemostasis assays. Thromb J 2014; 12: 24. [http://dx.doi.org/10.1186/1477-9560-12-24] [PMID: 25750588]

[32] Patel MR, Hellkamp AS, Lokhnygina Y, et al. Outcomes of discontinuing rivaroxaban compared with warfarin in patients with nonvalvular atrial fibrillation: analysis from the ROCKET AF trial (Rivaroxaban Once-Daily, Oral, Direct Factor Xa inhibition compared with vitamin K antagonism for prevention of stroke and embolism trial in atrial fibrillation). J Am Coll Cardiol 2013; 61(6): 651-8. [http://dx.doi.org/10.1016/j.jacc.2012.09.057] [PMID: 23391196]

[33] Granger CB, Lopes RD, Hanna M, et al. Clinical events after transitioning from apixaban vs. warfarin to warfarin at the end of the Apixaban for Reduction in Stroke and Other Thromboembolic Events in Atrial Fibrillation (ARISTOTLE) trial. Am Heart J 2015; 169(1): 25-30. [http://dx.doi.org/10.1016/j.ahj.2014.09.006] [PMID: 25497244]

[34] Eikelboom JW, Wallentin L, Connolly SJ, et al. Risk of bleeding with 2 doses of dabigatran compared with warfarin in older and younger patients with atrial fibrillation: an analysis of the randomized evaluation of long-term anticoagulant therapy (RE-LY) trial. Circulation 2011; 123(21): 2363-72. [http://dx.doi.org/10.1161/CIRCULATIONAHA.110.004747] [PMID: 21576658]

[35] Halperin JL, Hankey GJ, Wojdyla DM, et al. ROCKET AF Steering Committee and Investigators. Efficacy and safety of rivaroxaban compared with warfarin among elderly patients with nonvalvular atrial fibrillation in the Rivaroxaban once daily, oral, direct factor Xa inhibition compared with vitamin $\mathrm{K}$ antagonism for prevention of stroke and embolism trial in atrial fibrillation (ROCKET AF). Circulation 2014; 130(2): 138-46 [http://dx.doi.org/10.1161/CIRCULATIONAHA.113.005008] [PMID: 24895454]

[36] Dans AL, Connolly SJ, Wallentin L, et al. Concomitant use of antiplatelet therapy with dabigatran or warfarin in the Randomized Evaluation of Long-Term Anticoagulation Therapy (RE-LY) trial. Circulation 2013; 127(5): 634-40. [http://dx.doi.org/10.1161/CIRCULATIONAHA.112.115386] [PMID: 23271794] 
[37] Levy JH, Faraoni D, Spring JL, Douketis JD, Samama CM. Managing new oral anticoagulants in the perioperative and intensive care unit setting. Anesthesiology 2013; 118(6): 1466-74. [http://dx.doi.org/10.1097/ALN.0b013e318289bcba] [PMID: 23416382]

[38] Schellong SM, Haas S. Novel oral anticoagulants and their use in the perioperative setting. Anasthesiol Intensivmed Notfallmed Schmerzther 2012; 47(4): 266-72. [http://dx.doi.org/10.1055/s-0032-1310416] [PMID: 22504623]

[39] Schlitt A, Jámbor C, Spannagl M, Gogarten W, Schilling T, Zwissler B. The perioperative management of treatment with anticoagulants and platelet aggregation inhibitors. Dtsch Arztebl Int 2013; 110(31-32): 525-32. [PMID: 24069073]

[40] Ishihara H, Torii H, Imoto H, Oka F, Sadahiro H, Suzuki M. Intravenous thrombolysis with recombinant tissue plasminogen activator in a stroke patient treated with rivaroxaban. J Stroke Cerebrovasc Dis 2014; 23(10): e457-9. [http://dx.doi.org/10.1016/j.jstrokecerebrovasdis.2014.07.008] [PMID: 25280819]

[41] De Smedt A, De Raedt S, Nieboer K, De Keyser J, Brouns R. Intravenous thrombolysis with recombinant tissue plasminogen activator in a stroke patient treated with dabigatran. Cerebrovasc Dis 2010; 30(5): 533-4. [http://dx.doi.org/10.1159/000319886] [PMID: 20881383]

[42] Jauch EC, Saver JL, Adams HP Jr, et al. American Heart Association Stroke Council; Council on Cardiovascular Nursing; Council on Peripheral Vascular Disease; Council on Clinical Cardiology. Guidelines for the early management of patients with acute ischemic stroke: a guideline for healthcare professionals from the American Heart Association/American Stroke Association. Stroke 2013; 44(3): 870-947. [http://dx.doi.org/10.1161/STR.0b013e318284056a] [PMID: 23370205]

[43] Müller P, Topakian R, Sonnberger M, et al. Endovascular thrombectomy for acute ischemic stroke patients anticoagulated with dabigatran. Clin Neurol Neurosurg 2013; 115(10): 2257-9.

[http://dx.doi.org/10.1016/j.clineuro.2013.07.017] [PMID: 23921437]

[44] Kimura S, Ogata T, Fukae J, et al. Revascularization for acute ischemic stroke is safe for rivaroxaban users. J Stroke Cerebrovasc Dis 2014; 23(9): e427-31. [http://dx.doi.org/10.1016/j.jstrokecerebrovasdis.2014.05.015] [PMID: 25149204]

[45] Berkhemer OA, Fransen PS, Beumer D, et al. MR CLEAN Investigators. A randomized trial of intraarterial treatment for acute ischemic stroke. N Engl J Med 2015; 372(1): 11-20.

[http://dx.doi.org/10.1056/NEJMoa1411587] [PMID: 25517348]

[46] Saver JL, Goyal M, Bonafe A, et al. SWIFT PRIME Investigators. Stent-retriever thrombectomy after intravenous t-PA vs. t-PA alone in stroke. N Engl J Med 2015; 372(24): 2285-95.

[http://dx.doi.org/10.1056/NEJMoa1415061] [PMID: 25882376]

[47] Molina CA, Chamorro A, Rovira À, et al. REVASCAT: a randomized trial of revascularization with SOLITAIRE FR device vs. best medical therapy in the treatment of acute stroke due to anterior circulation large vessel occlusion presenting within eight-hours of symptom onset. Int $\mathrm{J}$ Stroke $2015 ; 10(4): 619-26$.

[http://dx.doi.org/10.1111/ijs.12157] [PMID: 24206399]

[48] Campbell BC, Mitchell PJ, Kleinig TJ, et al. EXTEND-IA Investigators. Endovascular therapy for ischemic stroke with perfusion-imaging selection. N Engl J Med 2015; 372(11): 1009-18. [http://dx.doi.org/10.1056/NEJMoa1414792] [PMID: 25671797]

[49] Goyal M, Demchuk AM, Menon BK, et al. Randomized assessment of rapid endovascular treatment of ischemic stroke. N Engl J Med 2015; 372(11): 1019-30.

[http://dx.doi.org/10.1056/NEJMoa1414905] [PMID: 25671798]

(C) Patel et al.; Licensee Bentham Open.

This is an open access article licensed under the terms of the Creative Commons Attribution-Non-Commercial 4.0 International Public License (CC BY-NC 4.0) (https://creativecommons.org/licenses/by-nc/4.0/legalcode), which permits unrestricted, non-commercial use, distribution and reproduction in any medium, provided the work is properly cited. 